\title{
Can dietary supplements maintain cognition in mid- to late-life?
}

\author{
Alexander L. W. Smith $(1)$ \\ COMMENTARY ON... COCHRANE CORNER ${ }^{\dagger}$
}

\begin{abstract}
SUMMARY
Cognitive health, and prevention of its decline to dementia, has risen in prominence with a corresponding exploration of modifiable risk factors to prevent a decline in cognitive health with age. This commentary discusses a new Cochrane review that examines the effect of vitamin and mineral supplementation in maintaining cognitive health in cognitively healthy adults in mid- and late-life. From a heterogeneous body of evidence, the quality of which ranged from very low to moderate, the review draws the conclusions of little or no benefit of supplements.
\end{abstract}

\section{DECLARATION OF INTEREST}

None.

\section{KEYWORDS}

Dementia; cognition; supplements.

Dementia places a large burden on healthcare systems around the world: in 2015 it was estimated to have cost US $\$ 818$ billion (1.1\% of global gross domestic product), with 50 million diagnosed with the syndrome and 10 million new cases a year. This is forecast to rise to 152 million cases in 2050 (World Health Organization 2019). A 1-year delay in onset would forecast 12 million fewer cases worldwide in 2050 (Winblad 2016) and with a case requiring residential care costing approximately $£ 2500$ per month in the UK (Gustavsson 2011), the societal and healthcare costs would be significant.

There is currently no cure for dementia and limited progress in therapeutics for the condition, and therefore an interest in modifiable risk factors throughout the lifespan has developed (Hussenoeder 2018). Some of this focus has involved vascular and dietary risk factors (Hill 2019). There is a growing body of literature linking nutrition, in particular lower levels of vitamins such as folate and vitamins $\mathrm{B}_{12}, \mathrm{C}$ and $\mathrm{E}$, and the development of dementia in the cognitively impaired (Gustafson 2015).

In terms of current guidance in the UK it is advised not to offer ginseng, vitamin $\mathrm{E}$ or herbal supplements to those already diagnosed with dementia (National Institute for Health and Care Excellence 2018), the National Health Service (NHS) advising a healthy diet as a modifiable risk factor for dementia (NHS Website 2017). There is little guidance relating to supplements in maintaining cognitive health in those not diagnosed with dementia, although the Scientific Advisory Committee Network concluded that overall there was insufficient evidence 'that higher intakes of individual nutrients protect against cognitive decline, MCI [mild cognitive impairment] or dementias, including $\mathrm{AD}$ [Alzheimer's disease]' (Scientific Advisory Committee on Nutrition 2018: p. 47). The World Health Organization strongly recommends that "Vitamins B and E, polyunsaturated fatty acids and multi-complex supplementation should not be recommended to reduce the risk of cognitive decline and/or dementia' (World Health Organization 2019: p. xii).

\section{The Cochrane review}

\section{Definition of the clinical question}

The Cochrane review by Rutjes et al (2018) featured in this month's Cochrane Corner aimed to evaluate the effects of vitamin and mineral supplements on cognitive function (i.e. their 'efficacy') in cognitively healthy people aged 40 years or older.

Specifically, the primary outcome (Box 1) was maintenance of mean overall cognitive functioning in cognitively healthy individuals in mid- and late-life, assessed using recognised measures of cognitive function. The authors acknowledged that the degree of change (or not) in scores that qualifies as maintenance is unknown and opted for maintenance being no statistical difference between intervention and control groups with a significance value of $P<0.05$.

The authors also measured nine secondary outcomes covering validated measures of specific subdomains of cognitive functioning (e.g. executive function, speed of processing and episodic memory), incidence of mild cognitive impairment or all-cause dementia, quality of life, mortality,
ROUND THE CORNER
Alexander L. W. Smith, BSc (Hons), MBBS, is currently a core trainee in Health Education England - Thames Valley, UK, with an interest in experimental medicine. Correspondence Dr Alexander Smith, Cotswold House, Warneford Hospital, Warneford Lane, Oxford OX3 7JX, UK. Email: Alexander. Smith@oxfordhealth.nhs.uk

First received 5 Jul 2019 Final revision 1 Oct 2019 Accepted 10 Oct 2019

\section{Copyright and usage} (C) The Author 2019

\section{${ }^{\dagger}$ See this issue}


BOX 1 Primary outcome - why it is important to state this beforehand

Stating outcomes is key to trial design and drawing conclusions for clinical practice. They are set by the investigators before commencement of a trial, usually on the basis of previous similar studies or consensus among the field.

It is key for design first to protect against a type 1 error (i.e. false positives) due to testing a sample for multiple outcomes. Second it would protect against a type 2 error (i.e. false negatives), by calculating the required sample size to confidently reject a null hypothesis.

If all outcomes were of equal importance a trial would need several sample sizes to confidently reject the null hypothesis for each outcome. This could lead to testing an unnecessarily large sample (which would prove more expensive in terms of finance, resource and participants) or too small a sample to confidently reject the null hypothesis, which can equally be classed as unethical and wasteful (Andrade 2015).

It is not always possible to state a primary outcome, for instance in exploratory trials, in which case caution should be exercised in the interpretation of results.

If it is not clear in a published trial what the primary outcome is and you feel it should be, a quick look on a clinical trial registry such as ClinicalTrials.gov may clarify. functional performance, number of participants experiencing an adverse event and clinical global impression. Although clinically important, these will not be the focus of this commentary, first because of the incomplete data available for each measure in each supplement category and second because the space available to me here precludes further consideration of this aspect of the review.

\section{Method}

The authors included randomised and quasi-randomised controlled studies in any language. Trials ranged from 3 months to up to 20 years in duration, with cognitively healthy (or at least $80 \%$ of the sample deemed cognitively healthy) participants aged 40 years and over. Interventions were pooled into six groups according to supplement/s: antioxidants; B vitamins; vitamin D and calcium; zinc and copper; selenium; and complex multivitamins. Control comparisons were interventions that were not expected to affect cognition (placebo or usual care). It could not be ascertained what usual care entailed specifically, as many individuals in midto later-life use multivitamins and some trials did not use placebo as a comparator. Since the exact mechanism of action of vitamins on cognition is yet to be elucidated, we cannot be confident that comparator interventions have no impact on cognition. This could affect results by minimising any difference seen between intervention and control groups.

The authors searched electronic databases and trial registries monthly and performed 6-monthly searches of a number of sources, including conference proceedings, for grey literature; they also screened the reference lists of trials and recent systematic reviews, and subject-specific guidelines, as well as contacting companies and experts in the field in an effort to obtain grey literature. This is relevant to the review's results, contributing to a wider evidence base and thus more precise results as well as reducing publication bias.

Measures of treatment effect used mean difference (MD) for continuous data-sets using the same scales and standardised mean difference (SMD) for continuous data using different scales (Box 2). Risk ratios were used for dichotomous data.

Risk of bias was assessed using the Cochrane's 'risk of bias' tool. Of the 28 studies included, 8 were at high risk of attrition bias and 6 at high risk of reporting bias, the latter largely attributable to the variable primary outcomes of the studies included, in some cases adding cognitive assessment at a later stage in the study. This is important as it could represent possible outcome reporting bias and, although post hoc analysis is acceptable when accounted for in design and analysis, inclusion of a test midway through a trial not designed appropriately for that test could lead to conclusions that are either inaccurate or have low strength.

The authors acknowledged the heterogeneity in the field and two of the statistics to measure it were $I^{2}$ and tau ${ }^{2}$.

No subgroup analyses (i.e. examining whether effect is different in different subgroups) were performed owing to the small number of trials included, and no sensitivity analyses (i.e. restricting analysis to one subgroup) were performed. Possible sensitivity analyses that could be valuable would be on subgroups with only cognitively healthy samples, or on those that measured cognitive function from the outset. These may have led to more precise results with higher certainty in the results.

\section{BOX 2 Mean difference and standardised mean difference}

\section{Mean difference}

Used in meta-analysis to group clinical trials that use the same scoring system for a continuous variable measuring the same illness, e.g. all studies that used the Mini-Mental State Examination to assess overall cognitive function.

\section{Standardised mean difference}

Often used in meta-analysis to group clinical trials that use different scoring systems for a continuous variable measuring the same illness, e.g. all studies that used the Telephone Interview for Cognitive Status and the MiniMental State Examination to assess overall cognitive function. 


\section{Results}

The 28 studies included gave a population of over 83000 participants aged 40 years and over who were deemed cognitively healthy (i.e. without a dementia diagnosis or cognitive impairment) at baseline. The definition was made by each trial separately, for example not having a formal dementia diagnosis, not scoring positive on the Telephone Interview for Cognitive Status (TICS) or scoring above a certain threshold on the Mini-Mental State Examination (MMSE). This variation in definition could have resulted in the inclusion of those with cognitive impairment not yet qualifying for a diagnosis of dementia. Despite wanting to examine cognitively healthy people, the authors included studies with at least $80 \%$ of the sample deemed cognitively healthy - it was not clear why this percentage was chosen but could it influence the outcome, for example by attenuating a small difference seen between cognitively impaired and healthy people. For example, for the hypothetical 20\% cognitively impaired in mid-life, it can be presumed that other stronger risk factors for cognitive impairment (e.g. genetic risks) may be present and therefore the efficacy of supplements in primary prevention could be underestimated. The authors comment that it is 'possible that some participants may not have been cognitively healthy at baseline'.

No benefit in overall cognitive function with B vitamin supplementation was observed at any time point. The quality of the evidence ranged from low to moderate. This finding is in agreement with a previous systematic review and meta-analysis in this area (Clarke 2014).

Antioxidant studies included three supplements (vitamins $\mathrm{E}$ and $\mathrm{C}$ and beta carotene). One study on vitamin $\mathrm{E}$ supplementation found a significative improvement in MMSE scores for the intervention group at 3-12 months $(\mathrm{MD}=1.4,95 \%$ CI $1.18-1.62, n=74)$. However, this finding is burdened by the very low quality of the evidence due to imprecision and indirectness (Box 3). Moreover, the clinical value of an improvement of 1.4 on the MMSE seems minimal in the context of advising an individual to take supplements for the rest of their life. It is possible that the MMSE is not sensitive enough to identify the cognitive changes examined here, although Tsoi et al (2015) found it to be of comparable sensitivity and specificity to alternative cognitive tests.

Only one study could be used to examine the effect vitamin C supplementation on overall cognitive functioning and this suggested that there may be a small beneficial effect after 5-10 years of treatment $(\mathrm{MD}=0.46$ (95\% CI $0.14-0.78, n$ not reported, $P=0.006)$. Beta-carotene showed small benefits in

\section{BOX 3 GRADE biases}

Imprecision: this can be related to having insufficient patients recruited or insufficient events occurring, which result in a wide confidence interval e.g. due to poor recruitment or inclusion of tests after a trial has been designed (in that power calculations for sample size were not sufficient for the test introduced)

Indirectness: as clearly stated by the GRADE handbook (Schünemann 2013), 'Direct evidence consists of research that directly compares the interventions which we are interested in, delivered to the populations in which we are interested, and measures the

outcomes important to patients'. Use of surrogate markers and indirect comparisons (e.g. $A$ v. C and B v. C, enabling A v. B conclusions) would be a source of indirectness.

Selection: systematic differences between the groups that are compared.

Attrition: withdrawals or exclusions of people entered into the study who share common characteristics compared with those that remain in the study (resulting in a difference in characteristics of the initial and ending samples). overall cognitive function (on the TICS) after an average of 18 years of treatment.

Two randomised controlled trials (RCTs) involving vitamin $\mathrm{D}$ supplementation were included but not pooled, owing to the fivefold difference in equivalent dose used between the studies. Additionally, one of the trials included calcium supplementation whereas the other (smaller) trial did not. The smaller trial, involving 60 participants, found no significant differences in Montreal Cognitive Assessment (MoCA) scores between the control and intervention groups ( $\mathrm{MD}=0.76$ points, CI not reported, $P=0.186$ ), with a moderate degree of certainty. The larger study $(n=4143)$ found no significant differences between placebo and control groups in terms of incidence of dementia or probable mild cognitive impairment over the 7.8 year follow-up (hazard ratio $\mathrm{HR}=$ $0.94,95 \%$ CI $0.72-1.24, P=0.68)$.

Only one RCT used zinc and copper supplementation and found no statistically significant effect on overall cognitive function (measured using the MMSE) after 5-10 years of supplementation $(\mathrm{MD}=0.6,95 \% \mathrm{CI}-0.19$ to $1.39, n=1072)$.

No significant difference was identified between placebo and selenium supplementation from a single arm of an RCT assessing incidence of dementia over a 12-year period $(\mathrm{HR}=0.83,95 \% \mathrm{CI}$ $0.61-1.13, P=0.23, n=7388$ ).

In terms of complex multivitamins (containing $B$ vitamins and antioxidants with or without minerals) no significant differences in overall cognitive functioning (measured using the TICS) were identified at either 2.5 years $(\mathrm{MD}=0.04,95 \% \mathrm{CI}-0.09$ to $0.18 ; n=5947)$ or 8.5 years $(\mathrm{MD}=0.12,95 \% \mathrm{CI}$ -0.14 to $0.38 ; n=2324$ ).

The quality of the evidence in all included trials was deemed to range from 'very low' to 'moderate' using the GRADE criteria. This is largely because 
of restriction of participants enrolled (e.g. samples were restricted by gender or comorbidity), leading to indirect conclusions and uncertainty regarding the risk of selection and attrition bias (Box 3). Additionally, the trials included were very heterogeneous in terms of design, duration and primary outcomes, and many potential problems can be identified, such as the possibility of including participants who were not cognitively healthy at baseline or who had no baseline cognition scores.

In terms of efficacy, the results were consistent in reporting no benefit of any supplement over the different time points. The confidence intervals were relatively small, which could indicate precise results, although it more likely reflects the limited number of studies included in each review component.

The duration of studies also varied considerably, with the review authors having to create arbitrary categories for classification of results/effects, which they acknowledged limited the pooling of studies for analysis (as evidenced by forest plots with one study per category). As the authors acknowledged, the value of this approach in terms of drawing confident clinical conclusions is questionable. However, it should also be acknowledged that the authors took efforts to bring some clarity and categorisation to a heterogeneous field.

\section{Discussion}

In summary, this review found insufficient evidence that giving supplements to cognitively healthy middle- or old-age adults had any effect on maintaining cognitive function or preventing dementia. Not only are the effect sizes small, but also their clinical relevance is questionable. Results for vitamin C could be drawn from only one study, with small effect sizes and a 95\% CI approaching zero. Bearing in mind the review authors' conclusion that this is low-certainty evidence, it shows that supplementation with vitamin $C$ is nevertheless unlikely to have a meaningful clinical effect on the prevention of cognitive decline.

The authors highlight that the evidence only indirectly addresses the clinical question with regard to doses and study duration. The lack of understanding of the relationship between cognition and vitamin/mineral supplementation possibly contributed to the wide variety of doses and duration of studies, which weakens the evidence of the field. However, the authors also suggest that grouping of such heterogeneous samples and interventions, especially in the studies of B vitamins, may disguise any subtle effects on cognition.

It is noteworthy that, although many studies did not examine cognitive status as a primary outcome, there were no comments on the feasibility and acceptability of taking supplements every day for several years, if not decades. Although the safety of over-the-counter supplements could be assumed in the short term, side-effects or adverse effects of any intervention/supplementation would warrant comment.

Cost-benefit analyses for supplementation, for example for vitamin $\mathrm{C}$ or beta-carotene compared with other interventions, would be interesting. The 2018 British National Formulary indicates that a month's supply of a vitamin B compound tablets would cost $£ 2.82$, while a month's supply of vitamin $\mathrm{C}$ at a dose for prophylaxis of scurvy (typical indication) would cost $£ 3.29$ (Joint Formulary Committee 2018). When crudely compared with other long-term medication, such as simvastatin (£0.52 for a month's supply) or ramipril ( $£ 0.81$ for a month’s supply), vitamin supplementation can appear relatively expensive.

Several problems affect the confidence one can have in the result of the review. First, most of the studies did not include cognition as a primary outcome and therefore did not perform baseline cognitive assessments or included assessments only at a later stage. Furthermore, the authors noted that, when used, the cognitive assessment often lacked the sensitivity to detect changes in cognition.

Second, samples were often restricted in ways (e.g. by gender or comorbidity) that limit applicability to older, clinical populations.

Third, incidence of dementia was assessed in only three included studies, so most of the results have only indirect implications for dementia prevention. The authors also noted that most studies assessing cognition were short term ( $<2$ years), which they felt was too short a period when evaluating mineral/ vitamin supplementation for the long-term prevention of mild cognitive impairment or dementia. It is thought that 5-20\% of over-65-year-olds have mild cognitive impairment. The annual rate of conversion from mild cognitive impairment to dementia is about 5\% (Alzheimer's Society 2015). In this context studies less than 2 years in duration are unlikely to identify cognitive deterioration and thus any impact vitamin supplementation would have on it.

\section{Implications for clinical practice and research}

With a hypothetical patient in clinic we can state that, based on current available evidence, there is no indication that using vitamins or supplements can maintain cognitive function or prevent dementia in the middle- and old-age population. Additionally, costs can become a burden, especially if bought 'over the counter', and there is some evidence suggesting that supplementation is not without risk. For 
instance, there is a risk of stomach cramp or diarrhoea with excessive vitamin $\mathrm{C}$ consumption and an elevated risk of lung cancer in smokers who consume more than the recommended daily vitamin C dose (Wooltorton 2003).

The implications for research include the questions that remain regarding doses, supplement combinations, characteristics of a suitable population and the duration of studies required to find any effects on cognition, in particular the long-term effects of supplements started in mid-life. Key to this will be the understanding of the pathology of cognitive decline and dementia and the role of vitamins/supplements in modifying it. The review authors suggest conducting smaller studies with highly sensitive cognitive assessments, akin to a phase II trial, to elucidate mechanisms and identify any subtle effects that supplementation may have.

As long-term studies involving vitamin $\mathrm{C}$ and beta carotene demonstrated potential effects, future research could focus on antioxidant vitamins (vitamin C and beta carotene) for maintaining cognitive health and/or as a modifiable risk factor for dementia.

\section{Acknowledgements}

I thank Dr Riccardo De Giorgi, Wellcome Trust Doctoral Training Fellow in the University of Oxford Department of Psychiatry for his comments and suggestions. The views expressed are those of the author and not necessarily those of the NHS or the Department of Health.

\section{References}

Alzheimer's Society Website (2015) Mild Cognitive Impairment (MCI). Alzheimer's Society (https://www.alzheimers.org.uk/about-dementia/ types-dementia/mild-cognitive-impairment-mci). Accessed July 2019.
Andrade C (2015) The primary outcome and its importance in clinical trials. Journal of Clinical Psychiatry, 76: e1320-3.

Clarke R, Bennett D, Parish S, et al (2014) Effects of homocysteine lowering with $B$ vitamins on cognitive aging: meta-analysis of 11 trials with cognitive data on 22,000 individuals. American Journal of Clinical Nutrition, 100: 657-66.

Gustafson DR, Clare Morris M, Scarmeas N, et al (2015) New perspectives on Alzheimer's disease and nutrition. Journal of Alzheimer's Disease, 46: 1111-27.

Gustavsson A, Brinck P, Bergvall N, et al (2011) Predictors of costs of care in Alzheimer's disease: a multinational sample of 1222 patients. Alzheimer's \& Dementia, 7: 318-27.

Hill E, Goodwill A, Gorelik A, et al (2019) Diet and biomarkers of Alzheimer's disease: a systematic review and meta-analysis. Neurobiology of Aging, 76: 45-52.

Hussenoeder F, Riedel-Heller S (2018) Primary prevention of dementia: from modifiable risk factors to a public brain health agenda? Social Psychiatry and Psychiatric Epidemiology, 53: 1289-301.

Joint Formulary Committee (2018) British National Formulary (BNF) 75: March-September 2018. BMJ Group \& Pharmaceutical Press.

National Institute for Health and Care Excellence (2018) Dementia: Assessment, Management and Support for People Living with Dementia and their Carers [NICE Guideline NG97]. NICE.

NHS Website (2017) Can dementia be prevented? Dementia guide. NHS (https://www.nhs.uk/conditions/dementia/dementia-prevention/). Accessed 7 August 2019.

Rutjes AW, Denton DA, Di Nisio M, et al (2018) Vitamin and mineral supplementation for maintaining cognitive function in cognitively healthy people in mid and late life. Cochrane Database of Systematic Reviews, 12: CD011906 (doi: 10.1002/14651858.CD011906.pub2).

Schünemann H, Brożek J, Guyatt G, et al (2013) Handbook for Grading the Quality of Evidence and the Strength of Recommendations Using the GRADE Approach (Updated October 2013). GRADE Working Group (https://gdt.gradepro.org/app/handbook/handbook.html).

Scientific Advisory Committee on Nutrition (2018) SACN Statement on Diet, Cognitive Impairment and Dementias. Public Health England.

Tsoi K, Chan J, Hirai HW, et al (2015) Cognitive tests to detect dementia: a systematic review and meta-analysis. JAMA Internal Medicine, 1450-8.

Winblad B, Amuyel P, Andrieu S, et al (2016) Defeating Alzheimer's disease and other dementias: a priority for European science and society. Lancet Neurology, 15: 455-532.

Wooltorton E (2003) Too much of a good thing? Toxic effects of vitamin and mineral supplements. Canadian Medical Association Journal, 169: 47-48.

World Health Organization (2019) Risk Reduction of Cognitive Decline and Dementia: WHO Guidelines. WHO. 